hardly ever can, to that of the numerals. I have argued in the memoir just mentioned, partly from this fact and partly because some of the number-forms twist and plunge and run out of sight in the strangest ways, unlike anything the child has ever seen, that these are his natural, self-developed lines of mnemonic thought, and are survivals of the earliest of his mental processes, and a clue to much that is individual in the constitution of his mind. I found that only about one in thirty adult males saw these forms, but suspected that they were more common in early life, and subsequently lost by disuse. This idea is abundantly confirmed by the returns of the Charterhouse boys. Nearly one in four has the habit of referring numbers to some visual mental form or other; often it is only a straight line, sometimes more elaborate. No doubt as the years go by, most of these will be wholly forgotten as useless and even cumbrous, but the rest will serve some useful turn in arithmetic and become fixed by long habit, and will gradually and insensibly develop themselves. For want of spact I must here close my statement of facts ; and, my data being thus imperfectly before the reader, it would be premature in me to generalise. I trust, however, that what has been adduced is enough to give a fair knowledge of the variability of the visualising faculty in the English male sex, and I hope that the examples of the use of my "Statistics by Intercomparison" will convince psychologists that the relative development of various mental qualities in different races admits of being pretty accurately defined.

Francis Galton.

\title{
II-THE UNITY OF THE ORGANIC INDIVIDUAL
}

I.

In the free exercise of our thought and volition, we would laugh to scorn the intimation that not in our own undivided personality are lodged these sovereign powers, but that they originate outside of it, dispersedly, within the diminutive lives of a vast number of microscopical threads and dots. We would resign our autonomy to the five or six billions of corpuscles composing our bodies, upon no other conditions than such as have convinced us that, in spite of all appearances to the contrary, the earth is moving round the sun. It constitutes the loftiest pride of our culture to abnegate subjective impressions in favour of the demonstrations of science. We know, therefore, how we shall have to deport ourselves in the presence of facts, if they visibly confront and obstinately oppose our feeling 
of centralised personality. The histologist spreads before us a shred of brain and a shred of muscle, and apparently there is nothing there but fibres and corpuscles. "These are the neural elements that compose your nervous system, these the contractile elements that make up your motor organs. It is they that feel and think, that move and overcome resistances. What you call the consciousness of your personality is nothing but a resultant of the activities of myriads of such separate beings, of which your so-called body is a mere aggregate." This way of looking at things, commonly adopted by men of science, cannot be ignored by philosophers, for it leads inexorably to certain definite philosophical conclusions. It admits of no compromise. If it is true, psychology will have unreservedly to bend to its bearings; and this is my plea for laying before a philosophical audience the somewhat biological contents of these pages. If sensations are in truth compounded from the data furnished by individual and elementary cells, then psychological results must inevitably be realised in a sphere transcending vitality. Our organisation will be nothing but an elaborate mechanical apparatus through which mysterious outside powers are keeping up a telegraphic communication with a realm of pure spirituality, and through which this spiritual realm reacts mechanically on the outside powers. To be logically driven to such a conclusion would be indeed a strange fate for a generation that takes both life and science seriously.

The Cell-theorists exult in- the idea that so many billions of separate beings are, unconsciously and nevertheless with rigorous precision, shaping and actuating the marvellous mechanism of our complex life. They do not perceive that their fancied configuration of elementary units would necessitate a complex system of supernaturally pre-arranged harmonies, compared to which the moving and harmonising contrivances of the 'Monadology' would appear simple and natural. It is rather humiliating for biologists to find out at the end that all their strenuous efforts to bring the phenomena of life within the sphere of physical and chemical explanation have only resulted in a most emphatic and powerful confirmation of metaphysical doctrines.

How was it possible for so many vigilant inquirers to persist in overlooking the necessary philosophical implications of their fundamental doctrine? No lack of serious and well-directed philosophical thought can be imputed to the foremost representatives of natural science. On the contrary, never before have its votaries been more earnestly striving to gain an understanding, not only of detached occurrences, but also of the general interdependence of natural phenomena. But never before, also, has the task of generalising been so utterly perplexing as just 
now. On the one hand, the growing consciousness of the ultimate mental consistency of all experience is rendering more and more suspicious and unsatisfactory the plain and definite tenets of the corpuscular theory of matter, tenets that have hitherto afforded so firm and safe a basis, at least for physical and chemical research. On the other hand, the extensive groundwork of verified and irrepressible facts, composing our scientific knowledge, makes it quite impracticable for impatient systematisers to palm upon us a view of things in any way incongruous with this imposing array of ascertained experience. The easy time is for ever past when natural events had merely to be explained so as approximately to tit a given system. We now find ourselves burdened with the harder task of having to construct a system that will accurately fit the natural events. There is placed before our minds a rich medley of seemingly disparate and yet somehow intimately interlacing facts; and now we wish to know how this scientific miscellany is to be conceived as the one consistent and uncontradictory whole which it unmistakably constitutes. Strict regularity is discovered wherever exact investigation penetrates, and yet however much we may be striving to reach a comprehensive view of nature, our bewildered understanding fails in the attempt; not, indeed, as might be supposed, because of the many gaps in our knowledge, but because of the irreconcilable contrasts in it.

All our numbering, weighing, measuring, and classifying does not suffice to lay effective hold on objects. We set out with the impression of having matter before us, and end with the conviction that it is all force; force not a twin-manifestation of materiality, keeping equal pace with it, but force encroaching on the very essence of matter, till nothing substantial is left. Force, then, under this aspect, would seem to disclose itself as the true reality, underlying material appearances. But as soon as we endeavour to seize upon it for close inspection, it evades our grasp, losing itself completely in its effects-transformed into something new and strange. It needs, indeed, only an eye keen enough to pierce beyond the veil of phenomenal repose to see it figured by the wildest imaginable phantasmagoria.

But however profound our diffidence may have become with regard to the ultimate consistency of existence, we are undoubtedly in possession of a stupendous stock of scientific truths gathered from the minute investigation of inorganic nature. Thus prepared and equipped, we approach the mystery of the organic world. We discover the very transition of the inorganic into the organic - the actual formation of organic compounds out of inorganic elements, and we trace a progressive qeries of synthetical products from the lowest to the highest 
organic substance. We cannot doubt that organic nature is the evolutional outgrowth of inorganic nature, and that the most complex organic product has been gradually elaborated from less complex compounds. But suddenly, amid this apparently satisfactory view of things, we are reminded of Berkeley's fundamental maxim - "To be is to be perceived"; and we realise the incontrovertible truth that the organism cannot possibly be the mere product of its inorganic factors, nor these inorganic factors themselves what they seemed to be, for their very nature and existence is conditioned by the perceiving mind which looks out from the organism that just before was considered to result simply from their own material combination and dynamical co-operation.

Thus in our progress from inorganic to organic nature, the scientific puzzle becomes still more tangled and diversified. If, on the one side, the material constituting organisms and the forces actuating this material are exclusively derived from inorganic sources, then animate existence is certainly altogether dependent on inanimate existence. If, on the other side, it is the specific vital activity of animate existences which endows inanimate existences with every one of their discernible qualities-which, indeed, renders them at all sensible realities, then surely lifeless nature is altogether dependent on living nature. The dilemma is inevitable. The consciousness of it now forms part of every thoughtful mind, haunting the certitude of first principles, and threatening with dissolution the entire subsistence of material as well as ideal reality.

We have to make clear to ourselves that it is a perfectly legitimate biological inference which has increased our scientific perplexity to such an alarming and overwhelming extent. It is our growing insight into vital activity that has dissipated objective as well as subjective substantiality. Modern scepticism is the unmitigated expression of a physiological fact. It is evident beyond dispute that the influences stimulating our organs of sense cannot possibly bear the least resemblance to the perceptions which they rouse in the brain, and which manifest themselves there organically, by dint of its own inherent vital powers and in conformity with its own indwelling vital laws. It was through the direct investigation of the biological phenomena of vision that Berkeley achieved his signal and decisive victory over hypostatical materiality. And it was at bottom also a physiological conception that led Hume to question the spontaneous efficacy of mind, and with it the innate inherence and necessary coherence of its ideas.

There remained then of the whole universe-unsustained by any material, mental, or spiritual support — the bare manifestation 
of a more or less coherent series of impressions, actual and remembered, only an evanescent play of loosely-bound phenomena, emerging for momentary use from vacant nothingness. Is this not truly the gist of Nominalistic Idealism, the muchvaunted philosophy of our age-life abandoned by reason, and delivered over to blind nihilism ?

Our thinking has, indeed, been caught in the giddy whirl of vitality, and now finds itself everywhere and nowhere at the same moment. The superficial contemplation of living activity has led it astray. The more profound contemplation of living activity will right it again.

It would seem as if Evolution contained a promise to life of fruitful toil, secured by enduring triumph and progressive power. But Evolution is as yet only empirically known in its general effects and collective workings. It is not scientifically intelligible as a power primordially directing life. The attempts at an application of the principles of Evolution to the fundamental properties of life have hitherto failed. It will presently be shown that neither Mr. Darwin's Pangenesis, nor Mr. Herbert Spencer's Polarigenesis, nor Haeckel's Perigenesis can stand the test of accurate inspection. The cell-theory is here everywhere in the way, creating impassable chasms between onganic gradations which evolutionally ought to follow each other in unbroken sequence and continuity.

However, it is in this direction, I believe, that we have to seek for a firm anchorage for our Philosophy, now adrift on a fathomless sea, and for ever severed from its traditional moorings.

\section{II.}

The great generalisation that, at the present moment, has gained unrestricted sway in biological research, may be shortly expressed in the following terms:-

The complex organisin is in reality an aggregate of morphologically distinguishable units. It is in these individual units that the fundamental properties of life are inherent, and the various activities manifested by the organism, as a whole, are resultants of the discrete activities of just so many of these antonomous elements as the organism is composed of.

It is clear that the biological inquirers, who accept this celltheory as ultimate truth, or, at least, as a final foundation for their science, do not profess to concern themselves with the real mystery of life. Nutrition, growth and reproduction, heredity and adaptation, motility and sensibility, in fact all the properties essential to living beings, are by them already presupposed as original endowments of the morphological units. 
Their task can only consist in showing how the living elements become associated and grouped so as to constitute a complex organism, and how, by their definite arrangement and co-operation, complicated functions are then performed. They keep in view, as far as possible, only the cycle of individual development, the ontogenetic circle, and are striving to establish within these limits a statical science, a science of definitely preestablished cell-multiplication and cell-association.

But, we may pertinently ask, is not the very emergence and maintenance of life essentially dynamical ? And is not even its visible embodiment a veritable vortex, only phenomenally stable, but in reality receiving shape and constitution from an unremitting flow of molecular change?

This fashioning of the form of life by molecular processes is obviously the one fact that, above all others, has to be investigated, if we seriously desire to become initiated into the secret - of life. We cannot accept the enigma of the cell as final. We are driven by the requirements of science to seek for an explanation of the primary properties of the living substance, nutrition, growth, reproduction, and all the rest.

The embryonic cell-structure, issuing from a single parent and resulting nevertheless in the strange diversity of tissues that compose the complex organism, is in itself an irrepressible plea for a strictly evolutional interpretation. It is perfectly legitimate to desire to know how vital properties, originally condensed within the compass of a single cell, become eventually unfolded, so as to be morphologically represented by specific tissues, occupying wide-spread regions of an organised commonwealth, composed of myriads of mere derivative daughter-cells. It would, indeed, be a triumph of biological insight, highly conducive to the formation of a real science of life, if we could possibly ascertain how the molecularly correlated energies of the initial morphological unit get so widely differentiated and dissevered, in the course of cell-multiplication, as ultimately to constitute the well-nigh exclusive properties of distinct groups of cells, the so-called tissues-muscular tissue ministering essentially to motility, nervous tissue to sensibility, the tissues of the entoderm to nutrition, and so on.

But there are vital changes that urge us still more peremptorily to inquire into the conditions that underlie organic plasticity and development. The variation of individual organisms from their generic type is a phenomenon that cannot evade our daily observation. Here the deviation and adaptation of vital forms and energies become immediately perceptible, and the scientific mind is impelled to account for such changes.

It is plain that if the shapes and functions of life are, indeed, 
not immutably fixed, if, moreover, such variations as occur do not entirely depend on intrinsic powers, but are, on the contrary, also conditioned by external circumstances, then surely such modifying external influences can be exerted only on the ultimate constitution of the morphological units. The specific motions that make up the life of the elements are those that must be affected by the modifying process; for the life of the entire organism is avowedly only the resultant of the lives of its elements. We find ourselves, therefore, again compelled to penetrate beyond morphological appearances into the secret nature of the cell itself. In order to explain any change that may be effected in the organism by influences emanating from the medium, we must question the molecular processes through which the change is necessarily realised.

An hypothesis having for its foundation the absolute invariability of organisms, would be too strikingly at variance with most evident facts to have a chance of being propounded by anyone. On the other hand, the variation and development of organisms solely by dint of intrinsic energies is a supposition utterly opposed to our most fundamental scientific conceptions; spontaneous change, under whatever guise, being inconceivable to our mind as now constituted.

The modification of organisms by external agents, expressing -as everyone will admit - an incontestable truth of nature, the exigencies of science make it imperative on us to inquire into the positive manner and means by which the constitution and the properties of the morphological units become altered through the action of external forces. This investigation involves necessarily an examination of all the fandamental properties of life, for we cannot proceed very far in our research without becoming aware that all vital properties are mutually interdependent, so that it would be an unprofitable effort to try to gain a separate understanding of any single one of them. We have then to set to work to find out with some degree of precision how the activities of life are connected with each other at their common source, the cellular protoplasm; and it is incumbent on biology not to disavow the task, however difficult it may seem. All complex organisms are said to be aggregates of morphological units; all phenomena of life, resultants of the peculiar energies of these units. Forces emanating from external sources induce changes in organic forms and functions. The effect of these modifying forces can be wrought only on the intimate structure and properties of the efficient elements. Biology is the science of organic forms and changes. Consequently, no essential progress can be made in that science without a more perfect insight 
into the innermost nature of the units, by which all organic shaping and changing is directed and executed.

I have purposely dwelt so long on the necessity of turning our attention to the study of the molecular motions that constitute the life of the morphological units, because to many prominent investigators the undertaking appears still visionary, or, at least, premature. I hope, however, to convince all candid judges, who may find time and inclination to follow my statements, that the endeavour is by no means hopeless, but that, on the contrary, it may be pursued with signal profit and success. Instead of deriding as intruders into a field of fruitless speculation those bold scientific pioneers who first ventured to penetrate to the remote starting-point of vital changes, we may well feel deeply indebted to them for having pointed out, and made accessible to us, a mine of real and as yet unappropriated wealth.

When we examine the sundry attempts at a scientific elucidation of the fundamental properties of life, as energies inhering in the morphological units, we invariably find that this ultimate research into vital activities is guided by one predominant principle of Aggregation. Here, as everywhere, from the building up of the molecule to the building up of the cell and the building up of the entire organism, the grouping of elements and composition of elementary forces is the one answer ever offered by our science in explanation of any fragment of the great worldriddle - the creation of an infinitely diversified yet coherent and consistent universe within the unitary consciousness of the organic individual.

The problem, as hitherto conceived by the few illustrious thinkers who have grappled with it, consists in the representation in thought of an organic molecule, which is the bearer of such properties as will enable it to construct the organism by aggregation in directions resulting from its own intrinsic forces; and which will enable it also to effect all vital movements by the composition of its own energies with the energies of the other aggregated molecules. All modifications are thus directly wrought on the constitution of these ultimate vital molecules, and are then secondarily expressed and magnified in the altered construction of the entire organism, and in the modified display of its functions.

Before scrutinising the special suppositions connected with this view of organisation, I will mention that, by diligent and long-continued observation of protoplasmic units, I have been led to look for the solution of the problem in an entirely different direction.

I would oppose to the aggregational view of organisation a segregational view, holding that the morphological subdivisions 
in the centralised organism are caused by the specification of a single protoplasmic individual, and not by the association of a multitude of such individuals. The current theory, according to which the complex animal organism comes into existence through the aggregation of a vast number of autonomous elements, I wish to supplant by a theory which strives to demonstrate that the complex animal organism is itself a unit, only partially specified into anatomical and histological provinces. This truth, so completely at variance with our present biological conceptions, has been irresistibly forced upon me during my protoplasmic studies, and this in spite of the utmost watchfulness against anything that might at all resemble Conceptualism or Platonic Realism.

The distinct morphological divisions of higher animals are, indeed, integrant, not constituent, parts. They are specialised and segregated from a pre-existing whole, and are in no way discrete and independent units joined together in the composition of a complex totàlity. Here, for once, we actually meet in nature with that peculiar order of precedence, which-introspectively projected-has inspired so many intuitional world-conceptions. The whole is here in all reality antecedent to its parts. The organism is prior to its tissues, the tissues prior to their supposed elements. The centralised organism is not, as universally essumed, a multiple of ultimate units, but is, on the contrary, itself one single individuality. It is resolvable neither into morphological nor into physiological elements, but remains from first to last one indivisible chemical integral, a monadic molecule of such stupendous vastness and complexity, yet withal so potent in the exact maintenance of its specific individuality, that our imagination faints in the effort to picture it.

Conscious of the immense amount and diversity of evidence that, during these last forty years of busiest scientific devotion, has been amassed by a host of enthusiastic workers in support of the Cell-theory; filled also with admiration and reverence towards the great masters who have formulated this luminous conception as the highest expression of our scientific insight into the solemn problem of life; it was only after many years of silent work had matured my conviction of its fallacies, and had supplied me-as I believe - with a far more adequate and profound view, that I have taken courage to lay before my fellowworkers the grounds and results of $\mathrm{my}$ dissent.

\section{III.}

Whence the centralised vitality and co-operative activity of the complex animal organism? How amidst the maze of myriads 
of merely agglutinated morphological elements is one ever to reach the efficient focus, where molecular forces are weaving the form and functions of organised beings?

Mr. Darwin, by the perfect restraint which the consciousness of real and assumed facts imposes upon his rigorously scrupulous mind, finds himself debarred from the possibility of attempting a molecular interpretation of the phenomena of life. Since the modifications undergone by organisms take place simultaneously and diversely in a great number of morphologically and physiologically independent vital units, how does it come to pass that we find all these separate changes, at the end, united and adequately represented in the minute compass of a single cell, detached from some more or less out-of-the-way part of the vast cell-aggregate? This germinal ingathering of dispersed faculties is an organic conjuring-feat of so astounding a character, that even the renowned sagacity of our great reformer must have felt sorely taxed in the effort to discover anything like a natural and serious explanation of it. As matters actually stand, the bewildered biologist is simply compelled to imagine the sundry faculties, thus widely distributed among many discrete and disparate cells, as being severally and bodily collected from their scattered seats, and then somehow adroitly conveyed as into a single box. Whatever there may be found of mediæval grotesqueness in this more involutional than evolutional hypothesis is certainly not due to the predilections of its propounder, but solely to the exigencies of the occasion. Yangenesis is really the only hypothesis of vital phenomena compatible with the cell-theory. The propagation by inscrutable germs of the specific nature of each separate morphological unit seems-under the conditions of our present science-the only consistent way of accounting for the reproduction of old-established and newly-acquired qualities.

The-task is first to explain how all the morphological units of the complex organism come to be represented in the germ-cell, and, secondly, how during the reproduction of the complex oryanism all its morphological units come to be evolved again in due succession and distribution. These are the questions forced upon us by the cell-theory. Pangenesis answers them in the only manner in which they can at all be answered. It assumes a mysterious power, which accomplishes the gathering and selection of the necessary gemmules, and it assumes another mysterious power, to which is entrusted the orderly combination and evolution of the gemmules. This latter power is assisted by the equally mysterious fundamental vital properties, nutrition, growth and reproduction. Pangenesis is, indeed, the reductio ad absurdum of the cell-theory. It offers an explanation that explains nothing to a theory that can never be explained. 
But before speculating any further on the putting together of the composite organism, let us $d$ well for a little on the undivided nature of the morphological units, those elementary organisms in which the vital powers finally reside. Swedenborg believed the entire universe to exist in the shape of a monster-man, we lesser men representing vital units, it might be, in the big man's liver or spleen. Within the spleere of animal personality, the celltheory is an exact reproduction of this conception; only it is supported by a great array of palpable and plausible evidence.

When, however, with unbiassed mind we contemplate the marvellous complexity of organisation reached by avowedly unicellular animals, we cannot escape the conviction that to understand the secret of their vital properties and structural differentiations, would be equivalent to the understanding of the entire secret of vitality.

Take the ciliated infusoria, for example, the Paramaecium aurelia, everywhere procurable, and you will find here hardly any organically essential part missing. You have a cortical layer, and it is from this layer that in the higher animals all sensory organs and the nervous system originates. You have a contractile layer, and it is from this layer that all ectodermic muscles are developed. You have a circumscribed and definite mass of protoplasm ministering to food-rssimilation, and it is from this protoplasm that the entoderm of the so-called metazoa derives its origin. There is an oral and an aboral pole, a head and a tail, a mouth and an anus, a ventral and a dorsal aspect. There is a gullet conveying food near to the extreme end of the organism. There are depurative organs, with definite and farreaching channels. There is also enclosed in the granular protoplasm a mysterious mass of almost hyaline substance, called the nucleus. This lively and most specifically constituted cell or animalcule darts about in all directions, sensitive to the manifold conditions of its medium. If by any means one could penetrate the vital enigma of this one living unit, one might almost rest satisfied. At all events, it would be but a secondary task to puzzle out afterwards how by a peculiar juxtaposition of myriads of units endowed with such potencies a complex organism is formed, simulating in its entirety exactly the same specific traits that are already primitively present in every one of its constituent elements.

What, then, constitutes the life of the single cell-its nutrition, growth and reproduction, its shape, heredity and adaptation, its motility and sensibility? How are these fundamental properties of animal beings connected with the material by which they are manifested.

It was Mr. Herbert Spencer, who, under the sway of the doc- 
trine of Evolution, first made a serious effort to reach the ultimate seat of vitality. His circumspect and penetrating mind discerned the necessity of referring the plienomena of vital existence and development to the molecular processes occurring in the living substance. He recognised the great truth that life is essentially and primordially dynamical ; that its morphological appearances result from the play of vital motions. This leading principle of biology he endeavoured to elucidate in the following manner:We know that in a proper medium crystals will form. Some peculiar force inherent in the chemical molecules, that are the constituent units of such crystals, compels them to range themselves during solid aggregation in definite directions, and with definitely varying degrees of cohesion. The peculiar power here at work we call Polarity, merely to give it a name. Polarity then means the influence which certain molecules of the same kind exert on each other during unimpeded aggregation, so that by their conjunction a morphological unit results, specifically shaped, and having physical properties specifically varying in the sundry directions of its shape. Now it is clear, that the constituent molecules of the living substance must be immensely more complex than other molecules, much more complex even than the molecule of protein. Perhaps five or six protein-molecules, chemically linked together by phosphorus or sulphur, may represent the unit of the living substance. Units of such atounical complexity will be the bearers of highly impressible constitutions, in which changes, occurring in their environment, will become faithfully registered. Thus by exposure to different influences the molecules of the divers organisms have in the course of generical evolution become widely differentiated, each organism being now made up of units quite specific to itself, in the constitution of which units the result of the entire life-history, of the entire derivation, of the organism rests securely preserved. Concomitant with this constitutional registration of impressions there is developed the mysterious power of polarity, the same kind of power which was found to be the formative energy inherent in crystalloid molecules. The polarity of the chemical or physiological units of organisms evinces itself in the reproduction, during unimpeded aggregation of the complete adult organism. Each separate unit represents constitutionally the entire organism, and on aggregation with so many other like units as will equilibrate all their inwoven polarities, the form of the adult organism is gradually gained. The adult organism is the result and expression of the complete equilibration of the polarities inwoven during evolution into the constitution of the physiological units. It is thus that Mr. Herbert Spencer endesvours to actuate the processes ministering to organisation. 
And it must be confessed that, if life is, indeed, to be shut up in a molecule, that registers evolutional changes merely by intrinsic modifications, the conception can perhaps hardly be improved upon. Grant the existence of the physiological unit, and you have to do the best you can with the given material.

But calmly viewed in the light of established scientific principles the hypothesis runs riot. It upsets everything. It refuses to bend to mechanical laws, and it contradicts evolution.

Polarity conceived as a directing force inhering in chemical molecules is not mechanically interpretable. Of course sundry attempts in that direction have been made, of which mode of explanation Redtenbacher's "System of Dynamids" may be considered typical. It is impossible to derive from atoms, conceived as points or globules, a difference of physical properties in the different directions of an eggregate of such atoms. However, it is only necessary to assume that the atoms themselves are axial beings, and that they range themselves on aggregation in directions corresponding to their axes, in order to derive whatever shape and whatever cohesive differences we need. The artifice of quietly smuggling into our premisses whatever we wish to extract from them, is here very palpable. It seems to be thought that we can reduce the strangeness of a mystery ad libitum, simply by decreasing the size of its material embodiment.

It is, indeed, mechanically intelligible how a globule can result on aggregation from unimpeded forces inhering in molecules, but not how the specifically deviating shape of a crystal can thrust itself into existence. Here we have, evidently, something at work counteracting the natural tendency of cohesion.

It is, however, just possible to construct a mechanical hypothesis of crystallisation, by having recourse to extrinsically directing forces. We may, for instance, imagine that thermal inequalities obtaining between the solvent and the crystallising substance, due to changes in their mutual molecular relations and to their different specific heats, may possibly give rise to a system of heat-waves forming definite stereometrical configurations, in which dynamical moulds the petrifying material gets shaped.

But allowing polarity to remain an occult property of molecules, we find that, whilst crystalloid molecules aggregate in crystals, colloid molecules, on the contrary, invariably aggregate in globules, when unconstrained. Even salts crystallising in colloidal solutions are thereby kept within globular confinement, and hyaline organic tissues, such as, for instance, crystalline lens fibres, are seen on imbibition to be convertud into a number of calloidal globules. Polarity fails to build up specific colloidal

22 
shapes of the most elementary kind; much less will it prove efficient to urge organic molecules into the divers forms of morphological units, and then, leaping the bounds of morphological individuality, still retain sufficient power further to coerce the autonomous units into the matured shape of the adult organism.

But the incompetency of Polarigenesis is by no meang the greatest difficulty in the way of the hypothesis of physiological units. Physiological units have somehow to multiply, have, in fact, most prolifically to propagate, and are therefore necessarily supposed to mould foreign matter rapidly into their own specific kind. They are believed to be endowed with such gifts of fertility, as will enable them to convert outside material into a multitude of other physiological units just like themselves. Now, it is quite evident that, compared with this procreative achievement, Dr. Charlton Bastian's spontaneous generations are mere child's play; nay, even the special-creation hypothesis involves the lesser miracle. The evolutional toil of endless ages has, at last, succeeded in establishing the physiological unit of a mouse or an elephant. In its marvellously specific constitution are embodied all the divers and multitudinous characteristics of the organism to which it belongs. By dint of these laburiously inwrought faculties, it represents in itself the entire animal. To maintain its own exalted integrity against disintegrating influences requires already a stupendous exertion on its part. But more, many more, whole shoals of just such units being wanted for the construction of the vast animal aggregate, it is thought sufficient for this creative purpose to place random material in close proximity to some genealogically derived units. With implicit reliance on the efficacy of the procreative miracle, it-is expected that somehow the genealogical units will prove equal to the task of converting ever so much dead pabulum into their own living likeness. It will be conceded that this generative feat would be strangely at variance with the undeniable truth that "construed in terms of evolution, every kind of being is conceived as a product of modifications wrought by insensible gradations on a pre-existing kind of being."

Of course, it will be forthwith objected, that vast amounts of foreign matter are, nevertheless, constantly and most obviously transformed into living substance. Precisely so. But, how this is effected is, as yet, a secret, though I hope it will remain a secret no longer to those who may summon up patience enough to read these few pages. However, in any case, nothing can alter the decision, that, according to the above cited formula, the physiological unit can give rise to a new being only by modifications wrought by insensible gradations upon its own self, and that 
it cannot possibly convert pabulum into a dense swarm of its own kind by merely bathing in it.

It may appear rather strange that on so cardinal a point as the new production of the living unit I should have to defend the evolution-hypothesis against one of its very greatest promoters. But it serves to show how much the conception of life needs elucidation. We are all now consciously working on the plan of chemical substitution, on the evolutional plan; modifying and elaborating by degrees a common system.

Haeckel, who, ever since Darwin's awakening call, has been persistently labouring at the great trask of radically evolutionising the science of life, has likewise presented us with a profound view of fundamental vital activities. The consummate biological knowledge and dauntless philosophical zeal of the illustrious investigator render this gift invaluable to all who are striving to penetrate to that remotest retreat of vitality, where with ceaseless toil molecular forces are weaving the wondrous substance of life.

According to Haeckel, the substance of a moner is as uniform as that of a crystal ; for like a crystal the moner consists of an aggregate of equal molecules. Physically, chemically and physiologically every part of this homogeneous, living substance, or plasson, is endowed with all the properties of every other part, and is therefore capable of performing all the functions manifested by the entire moner. The molecule of the plasson is, like all other wolecules, merely a definite combination of certain qualitative atoms. Only it is exceedingly more complex in its present highly elaborated state than the molecule of any other substance. Its properties result entirely from its chemical and physical constitution, and are in no way otherwise superadded to it. Certain atoms combined in certain ways make up certain substances, possessing certain inherent properties. This is the essence of it all, there being nothing enduring in the world save atoms. These are eternal and invariable, and are endowed with eternal and invariable energies, both physical and psychical. Concomitant with the material grouping of atoms to molecules, and of molecules to variously aggregated substances, there proceeds a combination of the energies of the respective atoms into resultant energies. The highest resultant of the kind makes itself known as the psychical energy of the higher animals. But even the least complex chemical process cannot be understood without ascribing sensation and volition to the atoms that take part in it. How could they otherwise exert their singular faculty of selective attraction? The living molecule, or plastidule, besides the properties which it has in common with other molecules, displays one energy peculiar to itself, and constituting the 
essence of its vitality. It possesses, namely, memory of an unconscious kind, or the power of reproducing its own specific motion, together with such modifications as may have been wrought upon it by external influences. By dint of this faculty of intrinsic reproduction, it not only maintains its own marvellously complicated identity, but it propagates also its specific motion to the adjoining pabulum, which, thus coerced into like atomic vibrations, is thereby also transformed into plastidules of the same kind. In this manner organic propagation, a faculty believed hitherto to constitute the most inscrutable of all vital manifestations, receives a mechanical interpretation. An organism propagates its own kind merely because its ultimate molecules convert foreign material into their own likeness, by transmitting their specific motion to it. Propagation of kind is therefore, in the last instance, propagation of specific motion. With the understanding of this most fundamental vital activity, all other manifestations of life become readily intelligible. For example, we can easily conceive how growth must take place under such conditions. There is, indeed, no essential difference between the growth of a morphological unit or plastide, and the growth of a crystal. The crystal grows by apposition, or extrinsic accretion of equal molecules. The plastide grows by interposition ${ }^{1}$ or intrinsic accretion of equal molecules. When by the addition of more and more plastidules the plastide has increased so much in bulk as to overreach the measure of its cohesive power, then division into two equal parts, or breaking up into many parts, or budding out of the plasson will occur. These well-known modes of multiplication openly displayed by the plastides are merely the visible result of the hidden propagation of plastidules.

Now it is clear, that two plastides or morphological nnits resulting from the division of a uniform mass of plasson, however equally endowed they may start on their separate courses, will eventually become exposed to different modifying influences. These influences will gradually change their respective plastidule-motions, thus differentiating the constitution of the two plastides. During this modifying process those plastidules which most readily adapt themselves to the changed conditions will by degrees convert all other plastidules, contained in the same morphological unit, into their own likeness, upon which the modification may be said to be thoroughly established. The plastide is then completely adapted. Now the plastide divides again, and again dissimilar influences cause further deviations from the parent-type. In this manner the differentiating opera-

1 The word generally used in this relation is "intussusception". However, it does not accurately express the process; and, besides, I have to reserve it for a much more significant use. 
tion proceeds from generation to generation, the whole series of diversified individuals representing together a many-branched genealogical tree. Each individual transmits faithfully to its offspring its own inherited motion as modified during its previous life-time. Thus by continual development from generation to generation new features are grafted on or rather are united with old traits, the last individual on the topmost twig of the evolutional tree representing the condensed result of all preceding changes.

This progressive generical differentiation is perfectly intelligible, the fundamental assumptions once granted. But now comes the difficulty. If, instead of following up the ideal ramifications in the development of separate units, derived from some primitive ancestor, with whom there has never existed any connexion in space, if, instead of contemplating such a phylogenetic tree, we fix our attention on the analogous ontogenetic tree, formed by the permanent association of a consecutive series of differentiated units, we shall find the puzzle becoming rather too much for us.

In vain have we at the start plausibly built up the vital molecule, have then derived from its multiplication the morphological unit, and then again from the multiplication of the latter an entire genealogical tree of successively differentiated individuals, and have at last constructed the complex organism by the permanent association of a complete succession of such diversified individuals; suddenly and inevitably we find ourselves here at the goal face to face again with the same old enigma of procreation. We commenced by assuming that we had conquered its secret at the very point at which it started into activity. We allowed as many plastidules as we needed to originate simply through propagation of the plastidule-motion. But now, after the propagating operation has unfolded itself to the utmost, we discover that the problem has, nevertheless, somehow evaded our penetration. Here, at the completion of the generative circuit, it confronts us again with unmitigated strangeness.

Through the propagation of plastidules the multi-cellular person was constructed. Now how is the propagation of this multi-cellular person effected? How are the diversely modified properties of its myriads of morphological units, at last, collected into a single reproductive element, the germ-cell? We have, positively, no clue as to how the plastidule of the germ-cell could have possibly registered all these miscellaneous and multitudinous impressions, so as to be fit to reproduce-by memory or anything else-the whole evolutional chain of units that makes up the complex organism.

At this stage of our inquiry we become aware of the wisdom 
of Pangenesis. It is, as I have already remarked, the only consistent complement of the cell-theory. In order to conceive the complex organism as made up of individual units, we have to assume that the germ-cell is a congeries of gemmules derived from every one of those units. But why, it will be asked, a congeries of growing and multiplying gemmules, and not at once a congeries of vltimate molecules ? Simply because, in order to evolve the multi-cellular organism from a single cell, we have to keep distinct all the divers potentialities, and we have also to keep intact the mystery of propagation.

It is the essence of the vital molecule, its raison $d$ etre and madus vivendi, to convert all pabulum, all adjacent assimilable stuff, into its own likeness. If we were then to crowd the germcell with all kinds of plastidules derived from the divers morphological units of the organism, forthwith the assimilative and propagative energy of all the plastidules would be set going. There would ensue such a turmoil and desperate struggle for existence among the throng as would ultimately destroy the least specific difference between the units. Peace would not be restored until complete equality had been established among them all. There would result a uniform plasson filling the germcell, representing an entirely new being, and fit to propagate only its own kind.

But not merely in its final outcome is Haeckel's system of Perigenesis self-destructive. Its fundamental assumption includes already as many scientific antagonisms as can be brought together. We have a molecule, the movements of which are actuated by memory, and which constitutes a circumscribed order of atomic vibrations that transmits all the details of its intrinsically involved motions and outside material, without ever diminishing thereby its own inhering source of energy. This in plain language means (1) that metaphysical spontaneity actuates motion; (2) that a body knocking against another substance, or throwing its shadow on it, can thereby transform the same into its own likeness; (3) that in vital units there occurs an ever-renewed creation of the most highly specific energies out of nothing. These are rather startling propositions to enter into the groundwork of a professedly mechanical hypothesis.

All this sufficiently proves how inextricably perplexed even the foremost biological thinkers are apt to become whenever they attempt to elucidate the fundamental properties of life in keeping with the cell-theory.

The specific plastidule-movement can signify nothing but the chemical constitution of the plastidule conceived in terms of motion. How such intrinsic motions, how the specific constitu- 
tion of any molecule can maintain itself amidst its colliding atoms in a multifariously resisting medium, is one of those mechanical puzzles as yet neglected by science or noticed only when materiality is altogether dissolved into modes of "motions. Then we are at the end of all our scientific jugglery, and face to face with nothing but a mystic world of woven motions, gorgeously spread in phenomenal repose, and safely poised on that one mathematical point, our present consciousness.

EDMUND MoNTGOMERY.

(To be continued.)

\section{III-ON THE FORMS OF LOGICAL PROPOSITION.}

Logicians have been much exercised in the attempt to determine the number and arrangement of the simple forms of proposition, and hardly any two who have reconsidered the question fur themselves seem to have agreed in their decision. If we were constructing a complete theory of Logic we should have to ask what is the true account, by which we should understand the most fundamental account, of the nature and import of a proposition, and on this point different accounts would be in direct hostility to one another. But when we are discussing methods rather than theories, this is not necessary. The question then becomes, which is the most convenient account rather than which is the most fundamental; and convenience is dependent upon circumstances, varying according to the particular purpose we have in view. For the present purposes of inquiry, there seem to be three different accounts of the import of a proposition; the ordinary or predication view, the class inclusion and exclusion view, and that which may be called the compartmental view. It may fairiy be maintained that one of these views must be more fundamental than the others, or possess a better psychological warrant, but it cannot be denied that they are all three tenable views; that is, that we may, if we please, interpret a proposition in accordance with any one of the three. We propose to inquire what are the prominent characteristics of each of these distinct, but not hostile, views. What are their relative advantages and disadvantages; to what arrangement and division of propositional forms do they respectively lead; and which of them must be adopted if we wish to carry out the design of securing the 